Evidence for Liquid-Liquid Phase Transitions in the Transition Metals

M. Ross, D. Errandonea, R. Boehler

February 29, 2008 
This document was prepared as an account of work sponsored by an agency of the United States government. Neither the United States government nor Lawrence Livermore National Security, LLC, nor any of their employees makes any warranty, expressed or implied, or assumes any legal liability or responsibility for the accuracy, completeness, or usefulness of any information, apparatus, product, or process disclosed, or represents that its use would not infringe privately owned rights. Reference herein to any specific commercial product, process, or service by trade name, trademark, manufacturer, or otherwise does not necessarily constitute or imply its endorsement, recommendation, or favoring by the United States government or Lawrence Livermore National Security, LLC. The views and opinions of authors expressed herein do not necessarily state or reflect those of the United States government or Lawrence Livermore National Security, LLC, and shall not be used for advertising or product endorsement purposes.

This work performed under the auspices of the U.S. Department of Energy by Lawrence Livermore National Laboratory under Contract DE-AC52-07NA27344. 


\title{
Evidence for Liquid-Liquid Phase Transitions in the Transition Metals
}

\author{
Marvin Ross ${ }^{1}$, Daniel Errandonea ${ }^{2}$ and Reinhard Boehler ${ }^{3}$ \\ ${ }^{1}$ Lawrence Livermore National Laboratory, University of California, Livermore, CA 94551 USA \\ 2MALTA Consolider Team, Departamento de Fisica Aplicada-ICMUV, Universitat de València, Edificio \\ de Investigación, c/Dr. Moliner 50, 46100 Burjassot (Valencia), Spain \\ ${ }^{3}$ Max Planck Institut für Chemie, Postfach 3060, D-55020 Mainz, Germany
}

In this paper we report the first experimental evidence for the existence of a liquid-liquid phase transition(LLPT) in the transition metals. The transition is evident from discontinuities in the melting slope measured at high pressure. The discovery of LLPTs constitutes the strongest evidence for the presence of polymorphic structures in transition metal liquids at high pressure, and confirms the thesis that they are responsible for the relatively low melting temperatures, low melting slopes, and unusual phase diagrams.

PACS number(s): 62.50, 64.70.Ja, 64.70.Dv

In earlier reports[1,2] we proposed that, in order to explain the apparent discrepancy between the diamond anvil and shock wave melting measurements of $\mathrm{Mo}$ and $\mathrm{Ta}, \mathrm{a}$ liquid-liquid phase transition(LLPT), or liquid-glass transition, had to be present in the phase diagram. LLPTs are characterized by liquids of the same composition, but with different densities, and different atomic configurations, often referred to as polymorphs. In this paper we present the first experimental evidence for LLPTs in transition metals. It is now well established that elements with partially filled $p$-electron valence shells have directional bonding and exhibit an LLPT. Some examples are carbon[3,4], silicon[5,6], nitrogen[7,8] and phosphorous[9,10]. Bellisent et al.[11] interpreted the structure of liquid As, obtained from neutron diffraction experiments, as the consequence of a strong Peierles distortion in the half-filled 3p-band[12].

The partially filled $d$-electron bands of transition metals also have the potential for a Peierles distortion, and directional bonding. The low melting slopes of transition metals has been attributed to directional $d$-electron bonding leading to the formation of 
preferred local structures with five-fold icosahedral or polytetrahedral short range order(ISRO) resulting from Peierls/Jahn-Teller(P/JT) distortions[12,13]. Evidence for the presence of ISROs in transition metal liquids is now well documented and are referenced in our earlier reports[1,2]. ISROs introduce geometric frustration inhibiting solidification, and their presence as soluble impurities increases the communal entropy, further favoring the liquid stability and lowering the freezing temperature. Since ISROs are denser than the pure atomic liquid, pressure enhances the concentration of local structures. Therefore, it should come as no surprise, that pure non-alloyed transition metal liquids, with partially filled $d$-bands, exhibit polymorphic behavior, and a pressure-induced LLPT.

The scarcity of experimental evidence for LLPTs in transition metals is at least partially due to the relatively high temperatures characteristic of these liquids. With the advent of the laser-heated diamond-anvil-cell technique it has now become possible to access melting curves of transition metals up to nearly $200 \mathrm{GPa}$ and $4000 \mathrm{~K}$. The experimental method employed on a wide variety of solids, including on the transition metals, has been described and referenced in earlier reports[14-17]. Melting is detected in a laser-heated diamond-anvil cell by warming a sample and observing the onset of speckle motion in the melt. Measurements are checked for reproducibility at least six times for every pressure point in each sample. We wish to emphasize that the laser speckle technique is very well suited to distinguish between solid-solid, and solidliquid transitions. When a solid-liquid transition is crossed, by increasing the laser power, speckle motion is maintained even when the laser power is held constant. On the other hand, when a solid-solid transition is crossed one observes a discontinuous, single event change in the laser speckle pattern.

Plotted in figures 1-4 are the melting measurements for a representative set of transition metals, $\mathrm{Cu}, \mathrm{Ni}, \mathrm{Ti}, \mathrm{Y}, \mathrm{Mo}$ and $\mathrm{Ta}$. With the exception of $\mathrm{Cu}$, all the transition metals studied, including those not included here, $\mathrm{Fe}, \mathrm{Co}, \mathrm{Cr}, \mathrm{V}$ and $\mathrm{W}$, exhibited an abrupt discontinuity in the melting slopes indicative of an LLPT. The sharpness suggests that the LLPTs may be first order-like, but masked by the uncertainty in the temperature. The experimental data for all these metals had been reported earlier[11- 
13]. However the presence of LLPTs was initially overlooked because of a reliance on data smoothing fitting functions.

$\mathrm{Cu}$ has a filled $3 d$-band, and a linear Lindemann-like melting curve and lacks any evidence of an LLPT that might result from P/JT distortions in the liquid. As such, $\mathrm{Cu}$ provides a useful reference system, or benchmark, for comparison with the other metals. Since, with few exceptions, transition metals do not undergo solid-solid phase changes along the melting curve, a sudden decrease in the melting slope implies an increase in the liquid density and an increase of the communal entropy, which may be attributed to an LLPT.

Figure 1 shows a plot of the $\mathrm{Cu}[17]$ and $\mathrm{Ni}[1,17-19]$ melting measurements, figure 2 shows $\mathrm{Cu}$ and $\mathrm{Ti}$, and figure 3 has $\mathrm{Y}[20]$. These data plots illustrate that $\mathrm{Ni}$, $\mathrm{Ti}$ and $\mathrm{Y}$ exhibit abrupt discontinuities in their melting curves which is indicative of an LLPT. In the case of $\mathrm{Ni}$ and $\mathrm{Cu}$ both have the same melting slopes up to $18 \mathrm{GPa}$. At this pressure, the Ni melting slope shows a sharp decrease. In the case of $\mathrm{Ti}$ and $\mathrm{Cu}$, at low pressure, they have very different melting slopes. This shows that at low pressure $\mathrm{Ni}$ has a relatively smaller concentration of ISROs and consequently behaves like $\mathrm{Cu}$. In contrast, Ti starts out with a significantly lower melting slope than $\mathrm{Cu}$ showing that it has a relatively larger concentration of ISROs than $\mathrm{Ni}$. Yttrium, which has a single $d$-electron has the narrowest $d$-band, and the sharpest discontinuity in its melting slope. This pattern of behavior is to be predictable, since the Jahn-Teller distortion favors the narrow bands of the early transition metals. Since $\mathrm{dT} / \mathrm{dP} \sim 0$ for $\mathrm{Ti}$ and $\mathrm{Y}$ at pressures above the transition, then $\Delta \mathrm{V} \sim 0$ and the new liquid phase is necessarily solid-like or glassy.

Some understanding of the ISROs in a liquid may be drawn from the work of Lee et. al.[21], who reported x-ray diffraction measurements of electrostatically levitated droplets of $\mathrm{Ni}$ and $\mathrm{Ti}$. From an analysis of the liquid structure factors they determined that the short-range order of liquid $\mathrm{Ni}$ is non-distorted icosahedral, while in $\mathrm{Ti}$ the icosahedral order is significantly distorted. Lee and Lee[22], employing first principles density functional calculations of $\mathrm{Ni}$ and Ti clusters, found a significant distortion of the Ti cluster icosahedron resulting in short bonds, but no meaningful distortion of the $\mathrm{Ni}$ 
icosahedron. Lee and Lee also found that the distortion in $\mathrm{Ti}$ becomes more severe when additional atoms are added to the $\mathrm{Ti}$ cluster creating larger clusters. The combined results of Lee et al.[21,22] are in agreement with the diffraction measurements and are consistent with the fact that the P/JT effect is stronger for the early metals [1].

In the case of Cs, which has $5 d$-electron character, and in a broad sense may be considered as a very early transition metal, Falconi et al.[23,24] have interpreted structural changes and density increases from x-ray diffraction patterns above 3.9 GPa that mark the change from a simple to a locally complex liquid. Their analysis "strongly suggests the existence of $d s p^{3}$ electronic hybridization", a P/JT distortion.

The melting curve measurements of Mo and Ta are shown in figure 4 . Both metals exhibit discontinuities in the melting slopes near 30-40 GPa implying the presence of LLPTs. The original measurements for Mo have been confirmed by Santamaria et al.[25] using x-ray diffraction techniques to detect melting, and for Ta by Errandonea et al.[26], also using x-ray diffraction and by Foata-Prestavoine et al. employing the speckle motion detection method[27]. Nevertheless, the DAC melting measurements, particularly the low melting slopes, have been controversial because of apparent disagreements with shock melting experiments of $\mathrm{Mo}[28,29]$ and $\mathrm{Ta}[30]$. In fact, the dynamic shock and static DAC measurements do not conflict, since they cover different regions of the phase diagram. In combination they provide new insights into the phase diagrams of transition metals [1,2]. We limit further discussion here to Mo, because the shock data for this metal is more complete, and in any case the properties of the Mo and Ta differ little.

Plotted for Mo, in figure 5, are the DAC melting measurements, the Hugoniot (dashed curve), and two data points, at $200 \mathrm{GPa}$ and $400 \mathrm{GPa}$, where discontinuities in the longitudinal sound speed have been detected in shock experiments. Hugoniot temperatures were calculated[29]. The shock discontinuity near $200 \mathrm{GPa}(\sim 4000 \mathrm{~K}$ has been interpreted as a bcc-hcp transition, and the discontinuity at $400 \mathrm{GPa}(\sim 10,000 \mathrm{~K})$ as melting from a solid to a liquid. Figure 5 includes a proposed LLPT involving an abrupt structural change between the phases; as referred to previously[2] as liquid and Phase II, and connecting the discontinuity in the Mo melting at $30 \mathrm{GPa}$ to the 400 
$\mathrm{GPa}(10,000 \mathrm{~K})$ shock transition. Near $400 \mathrm{GPa}$, the liquid is an ideal homogeneous fluid and Phase II is a phase in which long-range order is absent but pressure induces a high degree of local ordering.

Although, evidence is lacking to support the argument that the 200 GPa transition is bcc-hcp[2,31], Mo does in fact retain some shear strength along the Hugoniot in the regime between $200 \mathrm{GPa}$ and $400 \mathrm{GPa}$. In this case, liquid 2 would have had to undergo a continuous pressure-induced change starting from the liquid near $30 \mathrm{GPa}$ to a glasslike Phase II at $200 \mathrm{GPa}$. A linear extrapolation of the DAC melting curve to $200 \mathrm{GPa}$ predicts a melting temperature near $3330 \mathrm{~K}$, below the $4000 \mathrm{~K}$ calculated for the shock transition. This suggests that the shock sound speed discontinuity at $200 \mathrm{GPa}$ is from the bcc solid to a glassy state, and that at the 400 GPa transition the Hugoniot crosses the LLPT from a glass-like Phase II to a liquid. While very little theoretical effort has been directed to pressure-induced glass transformations, the parallel of a liquid to glass transformation studied by cooling can be instructive. For example, Doye et al.[32], employing a potential that favored the formation of five-fold coordinated liquid structures found that cooling lead to the formation of a polymeric network of linked icosahedrons.

An alternative interpretation of the Mo phase diagram, based mainly on computer simulations of the solid-liquid melting[33,34], discounts entirely the validity of the DAC measurements, and argues that a bcc-liquid melting curve connects directly from melting at 1 bar to the $400 \mathrm{GPa}$ shock discontinuity. However, these simulations treat the liquid as purely atomic (i.e hard-sphere or inert gas-like) and neglect the influence that locally preferred structures have on melting and a phase diagram with LLPTs.

The important new result in this report is the discovery of an LLPT in transition metal liquids. With exception of $\mathrm{Cu}$, the transition is ubiquitous having been detected in all of the ten transition metals so far studied. The presence of LLPTs provide the strongest evidence for the presence of polymorphic structures in transition metal liquids, and confirms the physical origin of the low melting temperatures, and low melting slopes measured in the diamond-anvil cell. 
Acknowledgements: The work by MR was performed under the auspices of the U.S. Department of Energy by Lawrence Livermore National Laboratory under Contract DEAC52-07NA27344. D.E. acknowledges the financial support of the Spanish MCyT. MR and D.E. also wish to thank the Max-Planck-Institute für Chemie at Mainz, Germany for its hospitality. 


\section{References}

[1] M. Ross, R. Boehler and D. Errandonea, Phys. Rev. B 76, 184117 (2007).

[2] M. Ross, D. Errandonea and R. Boehler, Phys. Rev. B 76, 184118 (2007).

[3] A. Ferraz and N.H. March, Phys. Chem. Liq. 8, 289(1979).

[4] O. Kum, F.H. Ree, S.J. Stuart and C.J. Wu, J. Chem. Phys. 119, 6053 (2003).

[5] N. Funamori and K. Tsuji, Phys. Rev. Lett. 88, 255508 (2002).

[6] N. Jakse and A. Pasturel, Phys. Rev. Lett. 99, 205702 (2007).

[7] M. Ross and F. Rogers, Phys. Rev. B 74, 024103 (2006).

[8] G. V. Mukherjee and R. Boehler, Phys. Rev. Lett. 99, 225701 (2007).

[9] Y. Katayama, T. Mizutani, W. Utsumi, O. Shimumura, M. Yamakata and K-I Funakoshi, Nature, 403, 170 (2000).

[10] T. Morishita, Phys. Rev. B 66, 054204 (2002).

[11] R. Bellisent, C. Bergman, R. Ceolin and J.P. Gaspard, Phys. Rev. Lett. 59, 661 (1987).

[12] R. Peierls, Quantum Theory of Solids (Pergamon, Oxford, 1955).

[13] H.A. Jahn and E. Teller, Proc. Soc. London, Ser. A 161, 220 (1937).

[14] R. Boehler, Nature, 363, 534(1993).

[15] D. Errandonea, B. Schwager, R. Ditz, C. Gessmann, R. Boehler and M. Ross, Phys. Rev. B 63, 132104 (2001).

[16] D. Errandonea, R. Boehler and M. Ross, Phys. Rev. B 65, 012108 (2002).

[17] S. Japel, R. Boehler, B. Schwager and M. Ross, Phys. Rev. Lett. 95, 167801 (2005).

[18] P. Lazor, G. Shen and S.K. Saxena, Phys. Chem. Minerals 20, 86 (1993).

[19] H.M. Strong and F.P. Bundy, Phys. Rev. 115, 278 (1959).

[20] D. Errandonea, R. Boehler and M. Ross, Phys. Rev. Lett. 85, 3444(2000).

[21] G.W. Lee, A.K. Gangopadhyay, K.F. Kelton, R.W. Hyers, T.J. Rathz, J.R. Rogers, and D.S. Robinson, Phys. Rev. Lett. 93, 1982 (2004). 
[22] B. Lee and G.W. Lee, J. Chem. Phys. 127, 164316 (2007).

[23] S. Falconi, L.F. Lundgaard, C. Jenny and M.I. McMahon, Phys. Rev. Lett. 94, 125507(2005).

[24] S. Falconi and G.J. Ackland, Phys. Rev. B 73, 184204 (2006).

[25] D. Santamaria, Personal communication.

[26] D. Errandonea, M. Somayazulu, D. Häusermann and D. Mao, J. Phy.:Condens. Matter 15, 7635 (2003); D. Errandonea, Physica B 357, 356 (2005).

[27] M. Foata-Prestavoine, G. Robert, M-H Nadal and S. Bernard, Phys. Rev. B 76, 104104 (2007).

[28] R.A. Hixson, D.A. Boness, J.W. Shaner and J.A. Moriarty, Phys. Rev. Lett. 62, 637 (1989).

[29] J.A. Moriarty, Phys. Rev. B 45, 2004 (1992).

[30] J.M. Brown and J.W. Shaner, Shock Waves in Condensed Matter, edited by J.R. Asay, R.A. Graham and G.K. Straub (Elsevier Science, New York, 1983).

[31] E.G. Moroni, G. Grimvall and T. Jarlborg, Phys. Rev. Lett. 76, 2758 (1996).

[32] J.P.K. Doye, D.J.K. Wales, F.H.M Zetterling and M. Dzugotov, J. Chem. Phys. 118, 2792 (2003).

[33] C. Cazorla, M.J. Gillan, S. Taioli, and D. Alfè, J. Chem. Phys., 126, 194502 (2007).

[34] A.B. Belonoshko, S.I. Simak, A.E. Kochetov, B, Johansson, L. Burakovsky, and D.L. Preston, Phys. Rev. Lett. 92, 195791 (2004). 
Figure captions

Fig. 1. $\mathrm{Cu}$ and Ni melting measurements. $\mathrm{Cu}$ (open circles)[17],

$\mathrm{Ni}($ filled circles)[1,17]. Ni(filled squares)[18]. Ni(filled triangles)[19].

Fig. 2. $\mathrm{Cu}$ and Ti melting measurements. $\mathrm{Cu}$ (open circles)[17], Ti(filled circles)[15].

Fig. 3. Y melting measurements.(filled circles)[20].

Fig. 4. Mo and Ta melting measurements, (empty circles)[14].

Fig.5. Mo phase diagram. DAC measurements(filled circles)[15], with extrapolation to $200 \mathrm{GPa}$ (long dashed curve). Hugoniot with calculated temperatures (short dashed curve)[29]. Pressures at which discontinuities in the longitudinal sound speeds were detected on the Hugoniot (filled squares with error bars)[28,29]. 
Figure 1

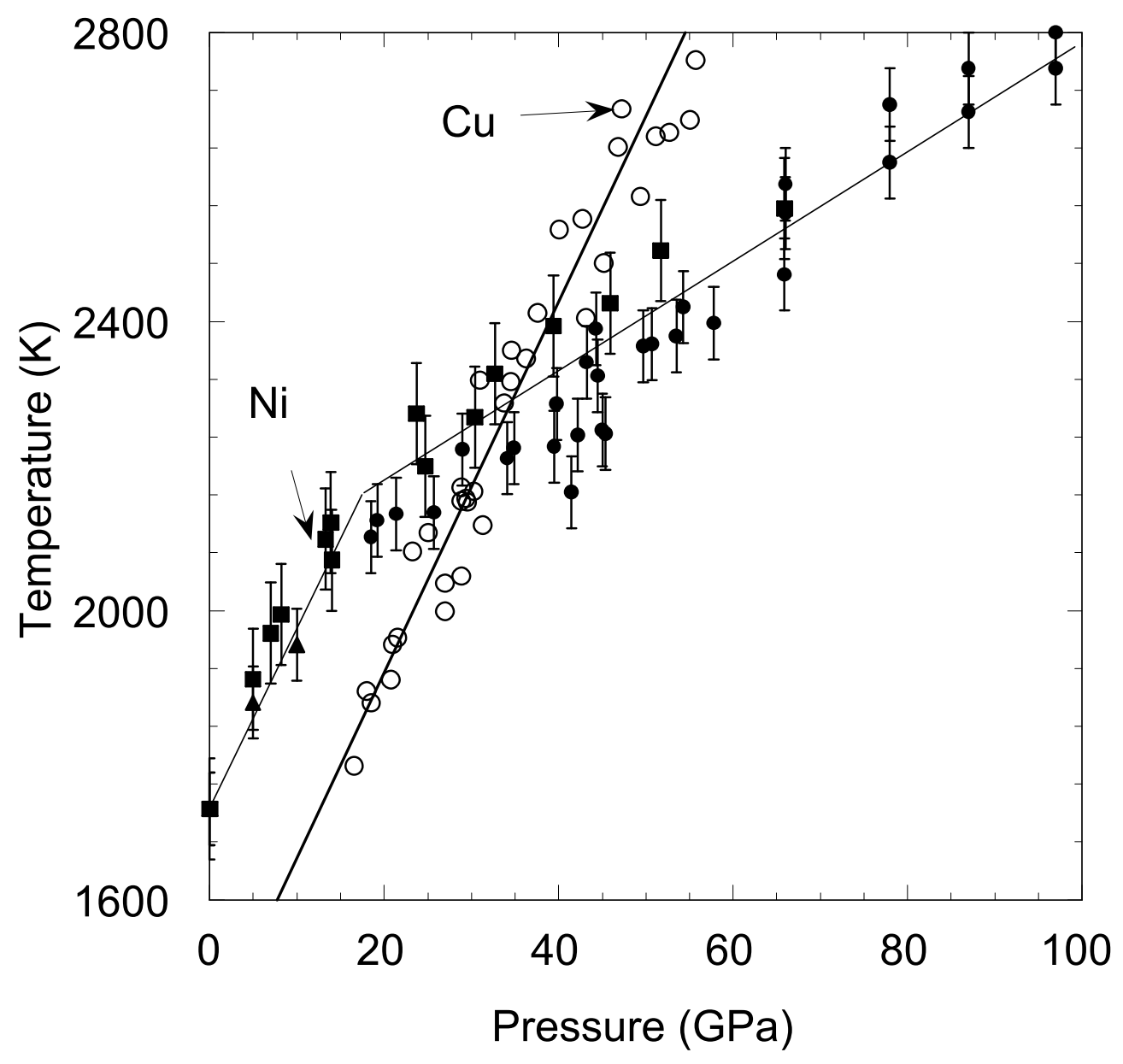


Figure 2

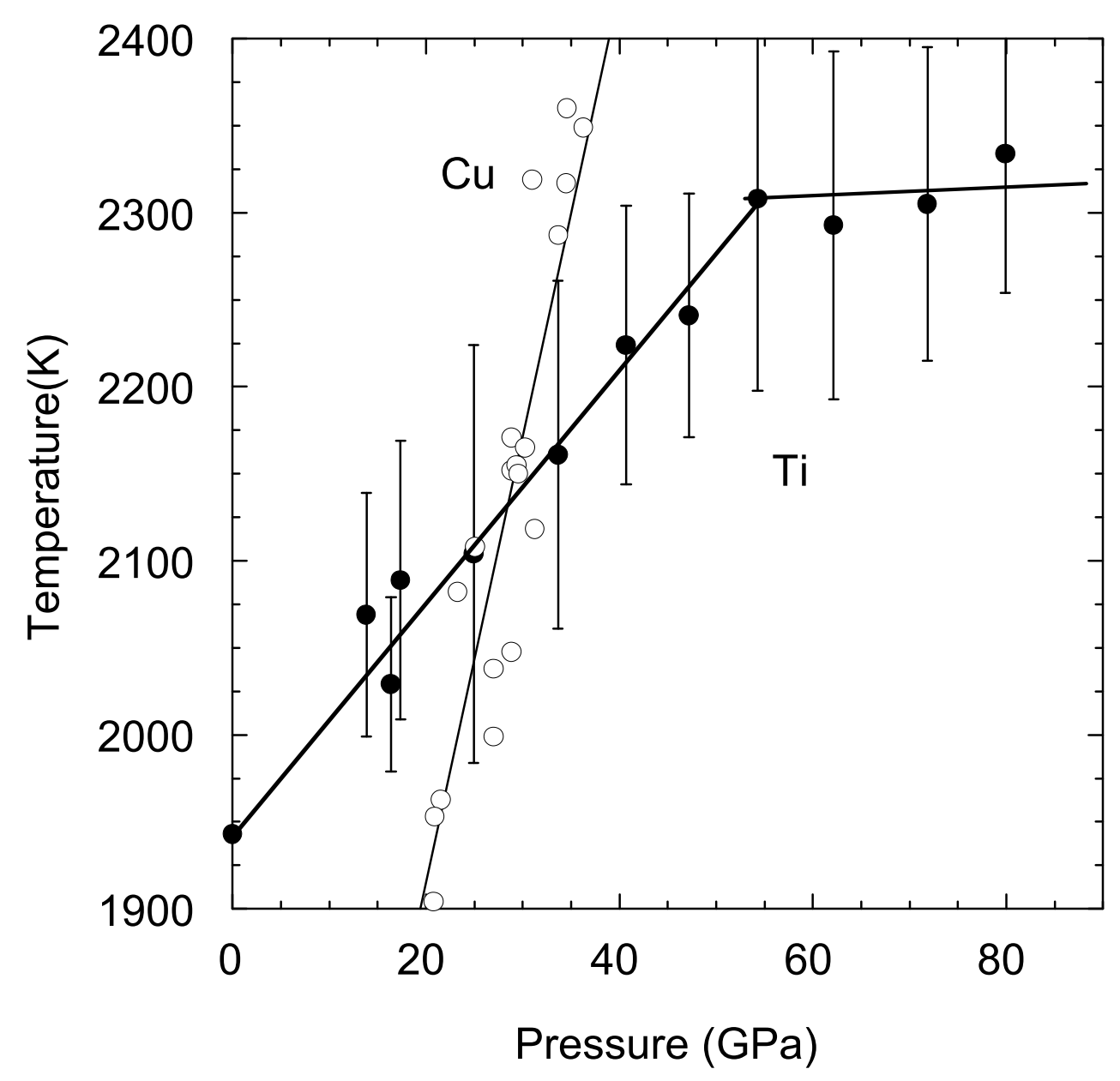


Figure 3

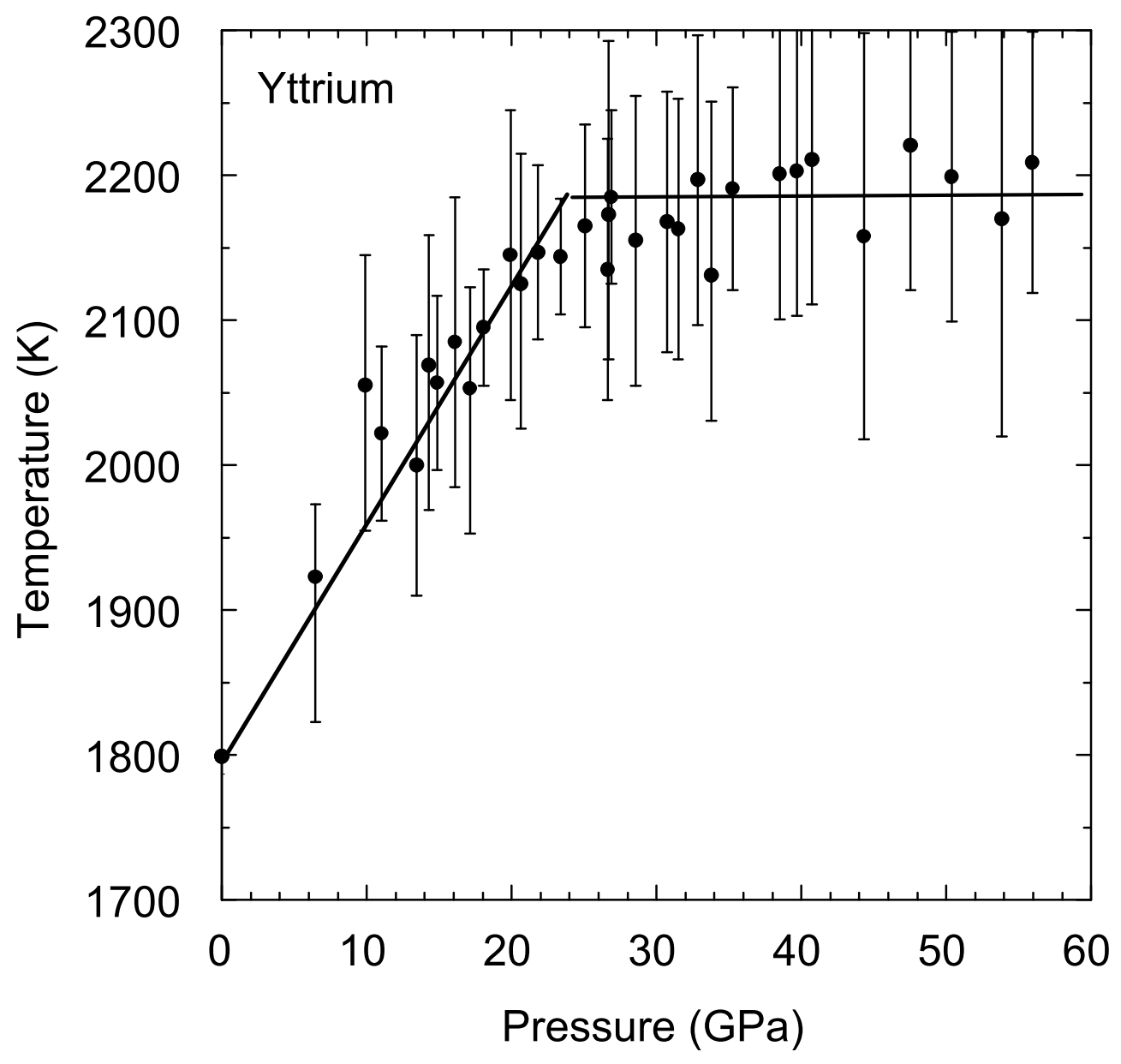


Figure 4

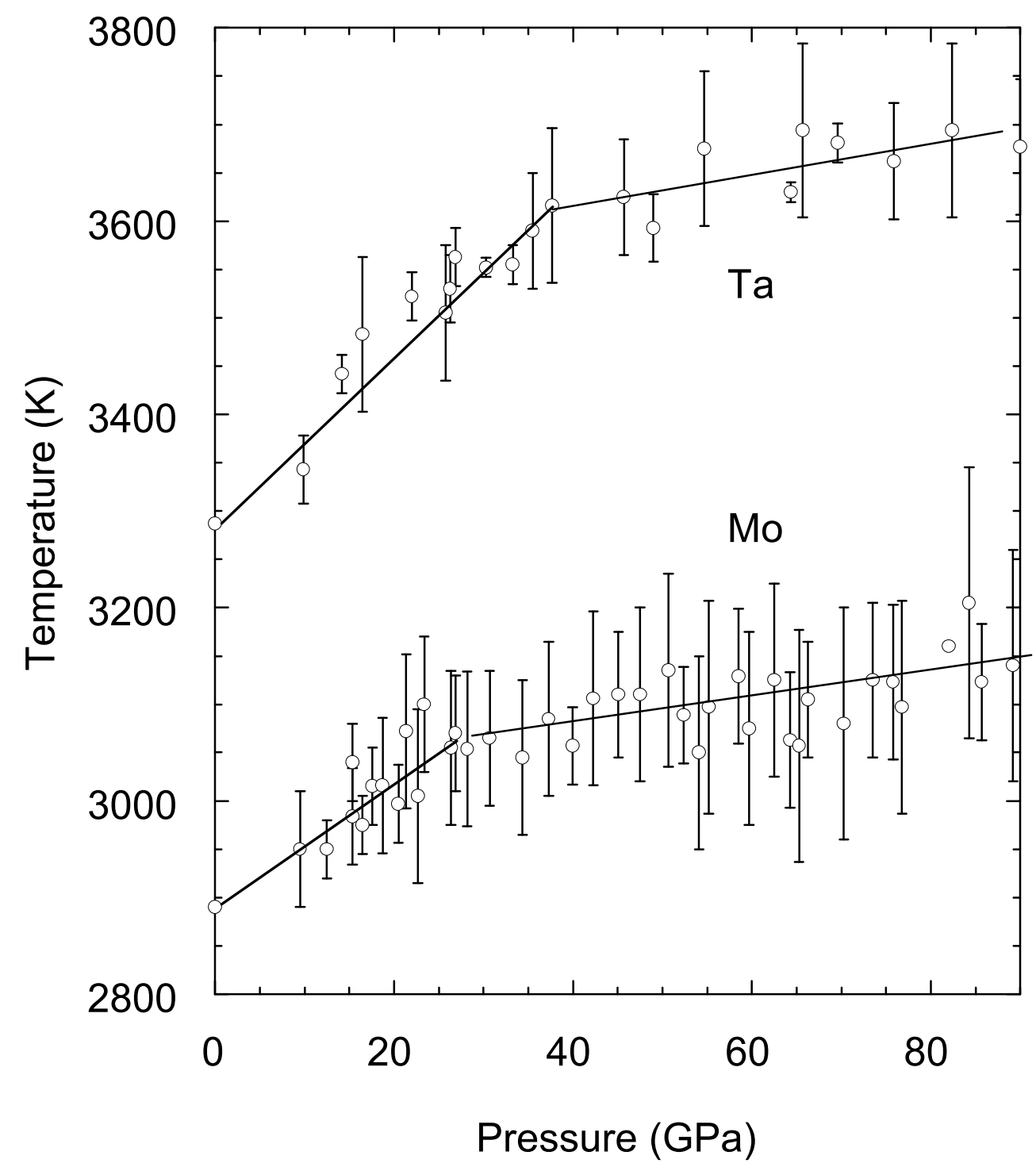


Figure 5

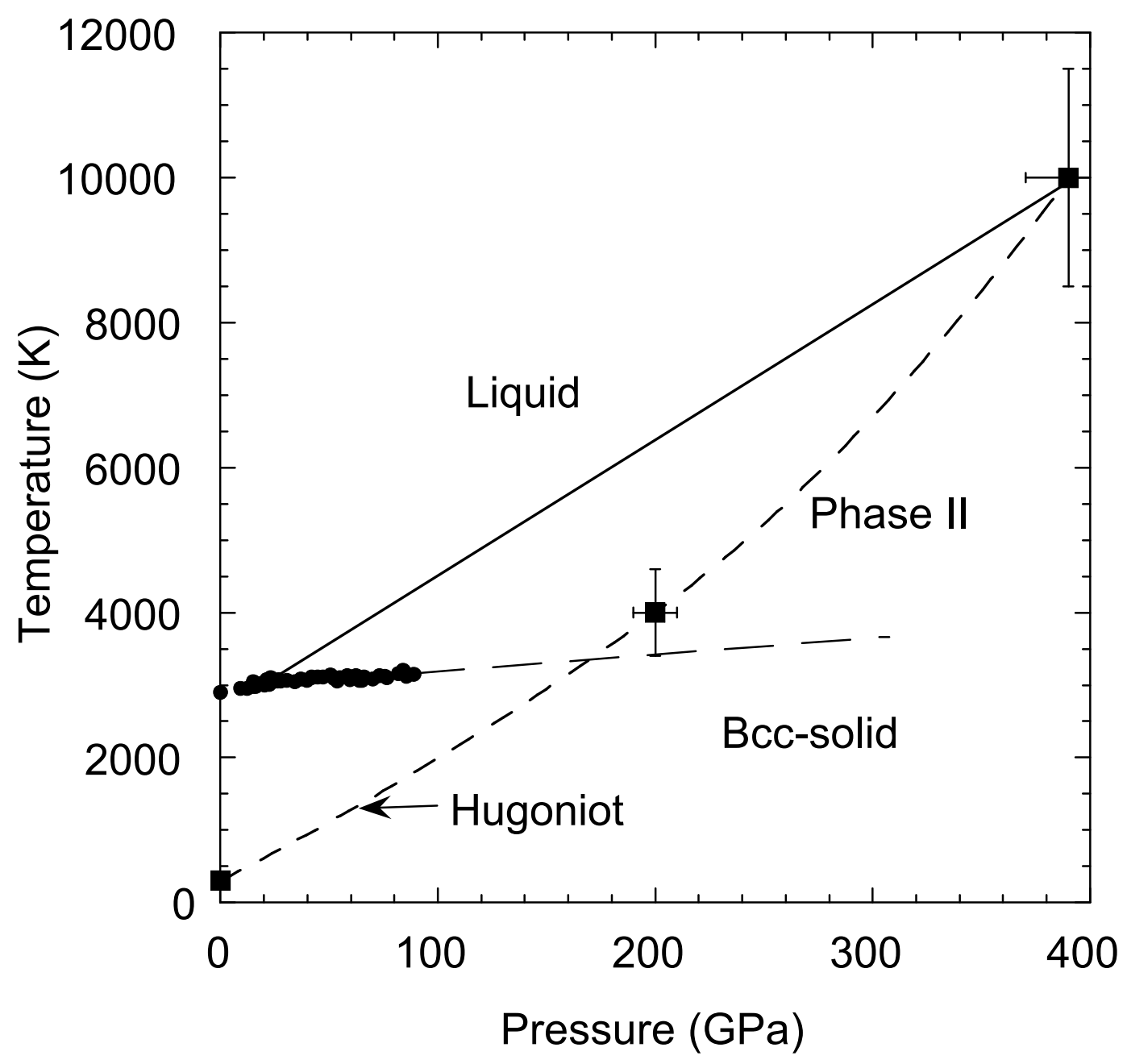

